\title{
Performance analysis of the MEDA's Thermal InfraRed Sensor (TIRS) on board the Mars 2020
}

\author{
Eduardo Sebastián, Joel Pérez, Andrés Bravo, Ricardo \\ Ferrándiz, Maite Fernández, J.A. Rodriguez Manfredi \\ Centro de Astrobiologia (CSIC-INTA) \\ Torrejón de Ardoz, Spain \\ sebastianme@cab.inta-csic.es
}

\author{
Antonio Peña, David González, Jose Moreno \\ Computadoras, Redes e Ingenieria S.A.U (CRISA) \\ Tres Cantos. Spain
}

\author{
Isabel Pérez-Grande, Adrián Chamorro \\ Universidad Politécnica de Madrid. IUM "Ignacio Da Riva" \\ Madrid, Spain
}

\begin{abstract}
The Thermal InfraRed Sensor (TIRS), an infrared (IR) radiometer developed at Centro de Astrobiologia, is one the sensors that compose the Mars Environmental Dynamics Analyzer (MEDA) onboard NASA's Mars 2020 mission. It will measure the net thermal infrared radiation, reflected solar radiation at the surface, atmosphere temperature and surface skin brightness temperature using five different channels. The present paper provides a brief description on TIRS design and channels requirements. Then, a detailed presentation of sensor model equations and a sensitivity analysis to model uncertainties are included. Finally, accuracy and resolution calculus for each channel versus operational temperature is presented. The calculus is performed based on sensitivity equations and the estimated values for different uncertainties sources.
\end{abstract}

Keywords-IR radiometer; Mars; Performance; Accuracy; Resolution; Requirements; MEDA: Mars 2020

\section{INTRODUCTION}

The Mars Environmental Dynamics Analyzer (MEDA) is one of the seven instruments onboard the Mars 2020 rover mission [1]. MEDA is a suite of sensors designed to characterize the near-surface climate of Mars and prepare for human exploration by assessing the environmental conditions across the rover traverse [2].

The Thermal Infrared Sensor (TIRS) is one of the six environmental sensors comprising MEDA [3].The TIRS has been designed to characterize the net thermal IR radiation at the surface, record the surface skin brightness temperature, the near-surface atmosphere temperature and the reflected visible an near infrared (VIS-NIR) solar irradiance. In combination with other MEDA's sensors, TIRS will allow the quantification of the surface energy budget and the determination of key geophysical properties of the terrain such as albedo and thermal inertia.

The thermal characterization of Martian atmosphere-surface boundary layer (PBL) has been done by instruments on board

The MEDA-TIRS is designed and built under funding of Instituto Nacional de Técuica Aeroespacial (INTA). Plan estatal de I+D+I (ESP201454256-C4-1-R, ESP2015-68281-C4-1-R, ESP2016-79612-C3-1-R) and CDTI.

\author{
Germán Martínez \\ University of Michigan, Atmospheric, Oceanic and Space \\ Sciences Department \\ Michigan, United States
}

\author{
Javier de Lucas, Pedro Hernandez \\ Centro de Metrologia y Calibración (INTA) \\ Torrejón de Ardoz, Spain
}

\author{
Miguel Ramos \\ Universidad de Alcalá, Departamento de Física y \\ Matematicas \\ Alcalá de Henares, Spain
}

of orbiters, like the IR camera THEMIS of the Mars Odyssey, or by in-situ measurements. Good examples are the rovers MERs with the spectrometer miniTES [4]. the contact sensor of the TECP probe contained in the Phoenix MECA instrument [5], or the REMS-GTS pyrometer on board rover Curiosity of Mars Science Laboratory (MSL) [6].

The principle of radiometric measurement of surface brightness temperature, or determination of soil mineralogy using thermopiles is a well proven concept which is applied at many space missions, e.g. at Thermal Mapper of the MultiPurpose Sensor (MUPUS-TM) on Rosetta Philae [7], the REMS-GTS on MSL mission, the MASCOT radiometer MARA of the Hayabusa II mission [8]. or the HP3-RAD radiometer for the NASA InSight mission to Mars [9].

Nevertheless. radiometers design for space applications implies challenges that occur with respect to the mass, size and power requirements. The necessity of operating in a remote and complex environment, form the thermal and dusty atmosphere points of view, are other important design considerations. Moreover, restrictions condition the usage of choppers or dust protection systems compromising radiometer performance $[10][9]$.

Radiometers end of life (EoL) accuracy and resolution performances are difficult to measure or compute. It is not for nothing there is a few paper showing these results. Nevertheless, any physical variable measurement must be accompanied by accuracy and resolution figures. An example of these limitations is the performance analysis done for the radiometer MARA. In [11] authors provide a theoretical analysis of radiometer resolution for each channel, considering only thermopiles' amplifiers noise. However. authors do not study other sources of noise such us thermopiles thermal noise or reference temperature amplifiers noise. Contrary in [8] accuracy and resolution at beginning of life $(\mathrm{BoL})$ are practically tested. using the calibration set up for functional or end to end test at different operational temperatures. 
As a general rule, instrument functional or calibration tests are not able to recreate or operate the system under extreme operational conditions, such us different thermal scenarios, sun irradiance or atmosphere composition, pressure and dusty conditions. Usually, tests provide performance data at instrument BoL, being quite difficult testing at EoL, since instrument ageing or degradation as result of the operation is quite hard to recreate.

The approach followed in this paper for TIRS performance analysis makes use of a sensor theoretical-practical model that is differentiated to obtain sensitivity equations. These equations relate a change or drift in any specific model variable or constant with an equivalent change in the incoming IR irradiance or temperature we want to retrieve. Thus, any radiometer uncertainty source, whatever the way to obtain its value (test or theoretical modeling and analysis of sensor and electronics) is introduced in the sensitivity equations to compute radiometer global accuracy and resolution figures.

\section{TIRS DESCRIPTION AND REQUIREMTNS}

TIRS design is an evolution of the REMS GTS on board Curiosity Rover of Mars Science Laboratory [10]. TIRS sensor head unit shall be accommodated on rover remote sensing mast looking forward at a height of $1.5 \mathrm{~m}$, while active electronics parts are located in the Mars 2020 rover body. Sensor head has an approximate weight of $97 \mathrm{~g}$ and a size of $58 \times 63 \times 58 \mathrm{~mm} \mathrm{[3].}$

TIRS IR transducers will be based on the thermopile TS100 from IPHT [12], with high heritage from REMS, MARA and HP3 instruments. Among thermopiles most important advantages are their capability of functioning at almost any temperature, small size, reduce cost, simple readout electronics, and sensitivity to all the IR spectra [13]. Thermopiles filter transmission bands have been selected to fulfill with the scientific purpose of each channel, see Table I. In the same way, channels pointing angles have been selected in agreement with scientific objectives as well as to avoid rover elements direct vision and to minimize rover thermal influence on the focused ground. The channels field of view (FoV) is fixed by design to $\pm 10^{\circ}$ and $\pm 20^{\circ}$ relative to pointing angles in the vertical and horizontal directions respectively.

TABLE I. TIRS CHANNELS DESCRIPTION

\begin{tabular}{|c|c|c|c|}
\hline Channel & Purpose & Filter band & Pointing angles \\
\hline IR1 & Downward LW & $6.5-30 \mu \mathrm{m}$ & Upward $\left(+35^{\circ}\right)$ \\
\hline IR2 & Air Temp & $14.5-15.5 \mu \mathrm{m}$ & Upward $\left(+35^{\circ}\right)$ \\
\hline IR3 & Upward SW & $0.3-3 \mu \mathrm{m}$ & Downward $\left(-35^{\circ}\right)$ \\
\hline IR4 & Upward LW & $6.5-30 \mu \mathrm{m}$ & Downward $\left(-35^{\circ}\right)$ \\
\hline IR5 & Ground Temp & $8-14 \mu \mathrm{m}$ & Downward $\left(-35^{\circ}\right)$ \\
\hline
\end{tabular}

TABLE II. TIRS CHANNELS REQUIREMENTS $(1 \sigma)$

\begin{tabular}{|c|c|c|c|}
\hline Channel & Dynamic range & Accuracy & Resolution \\
\hline$\left[\mathrm{R} 1^{\mathrm{ab}}\right.$ & $3.5-180 \mathrm{~W} / \mathrm{m}^{2}$ & $\pm(2.5+5 \%$ of the reading $) \mathrm{W} / \mathrm{m}^{2}$ & $\pm 0.9 \mathrm{~W} / \mathrm{m}^{2}$ \\
\hline IR2 & $173-293 \mathrm{~K}$ & $\pm 5 \mathrm{~K}$ & $\pm 1 \mathrm{~K}$ \\
\hline $\mathrm{IR}^{\mathrm{a}}$ & $0-230 \mathrm{~W} / \mathrm{m}^{2}$ & $\pm(1+5 \%$ of the reading $) \mathrm{W} / \mathrm{m}^{2}$ & $\pm 1.2 \mathrm{~W} / \mathrm{m}^{2}$ \\
\hline IR4 $4^{\mathrm{ab}}$ & $50-420 \mathrm{~W} / \mathrm{m}^{2}$ & $\pm(2+5 \%$ of the reading $) \mathrm{W} / \mathrm{m}^{2}$ & $\pm 2.1 \mathrm{~W} / \mathrm{m}^{2}$ \\
\hline IR5 & $173-293 \mathrm{~K}$ & $\pm 5 \mathrm{~K}$ & $\pm 1 \mathrm{~K}$ \\
\hline
\end{tabular}

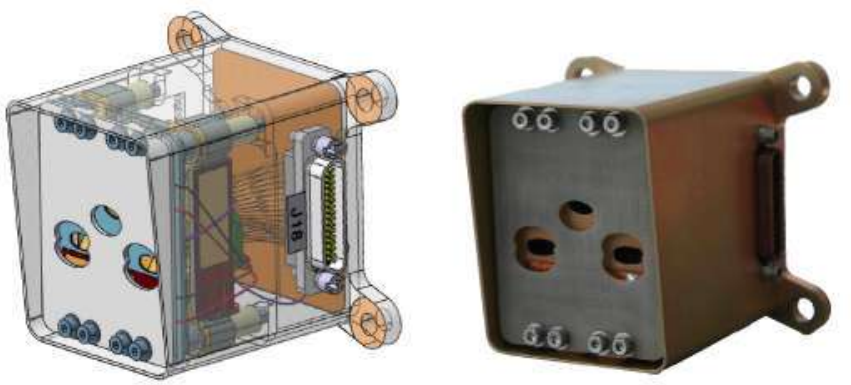

Fig. 1. TIRS 3D solid and bread board (BB) models

Thermopiles are located inside a mechanical structure denominated support plate that is thermally isolated from the TIRS housing, and protected from sun light direct incidence. This structure acts as a thermal inertia to stabilize detectors operating temperature, and thus increase the readout data accuracy. The support plate includes a couple of heaters that permits to keep constant detectors temperature during high performance operational mode. Its temperature is monitored by two resistance temperature detectors (RTD) that are used by heaters control system, as well as to estimate thermopiles' thermal gradients during in-flight calibration procedure [14] and the high performance mode.

Another important element in TIRS mechanical structure is the calibration plate, which is also used to the in-flight recalibration of some channels. This plate obstructs partially the thermopiles FoV, and making use of the two associated heaters, its temperature can be elevated during the calibration procedure. A RTD monitors its temperature and the readings are introduced in the target temperature or irradiance retrieval process.

Table II summarizes TIRS channels requirements in terms of dynamic range, accuracy and resolution. These requirements have been stablished based on: MEDA high level scientific objectives, TIRS expected performance, and seasonal and diurnal evolution of targets irradiances and temperatures, obtained from Martian atmospheric models and records [15][16].

\section{TIRS MODELING}

\section{A. Measurement Principal}

TIRS' thermopiles measure the net radiative flux exchanged between the sensing element or absorbing surface and the bodies around it: external as the target and calibration plate and internal as thermopile package inner surfaces [14]. The thermopiles' output voltage, $V_{\text {out }}$, is a representation of the temperature difference between the sensor package and the sensing element, which is in radiative balance with the target, and therefore influenced by its temperature or irradiance. Equation (1) shows the TIRS's channels mathematical model.

$$
\begin{aligned}
V_{\text {out }}= & S \cdot A_{d} \cdot F_{s-e} \cdot\left(\alpha \cdot \varphi_{t}+(1-\alpha) \cdot \varphi_{p}-\varphi_{c b}\right) \\
& +S \cdot A_{d} \cdot\left(1-F_{s-e}\right) \cdot\left(\sigma T_{c c}^{4}-\sigma T_{c b}^{4}\right)
\end{aligned}
$$

where: 
$S$ is the thermopile's responsivity in units V/W. It is a function of package temperature.

$A_{d}$ is thermopile absorber area in $\mathrm{m}^{2}$.

$F_{\text {see }}$ is thermopile external view factor.

$\alpha$ is the proportion of the thermopile external FoV covered by the target, while $1-\alpha$ is the one covered by calibration plate.

$\sigma$ is Stefan-Boltzmann constant.

$T_{c c}$ is the thermopile package lid temperature (front part).

$T_{o b}$ is the thermopile package socket temperature (rear part). acting as temperature reference.

$\varphi_{*}$ is the incoming target IR flux, or the solar reflected irradiance for channel IR 3 in $\mathrm{W} / \mathrm{m}^{2}$.

$\varphi_{p}$ is the incoming calibration plate IR flux in $\mathrm{W} / \mathrm{m}^{2}$.

$\varphi_{c b}$ is the leaving thermopile bolometer IR flux in $\mathrm{W} / \mathrm{m}^{2}$. follows:

IR fluxes are calculated according to Planck's law as

$$
\begin{gathered}
\varphi_{t}=\varepsilon_{t} \int_{0}^{\infty} \chi(\lambda) \frac{2 h c^{2}}{\lambda^{5}\left(e^{h c / \lambda K T_{t}}-1\right)} d \lambda \\
\varphi_{p}=\int_{0}^{\infty} \varepsilon_{p}(\lambda) \cdot \chi(\lambda) \frac{2 h c^{2}}{\lambda^{5}\left(e^{h c / \lambda K T_{p}}-1\right)} d \lambda \\
\varphi_{c b}=\int_{0}^{\infty} \chi(\lambda) \frac{2 h c^{2}}{\lambda^{5}\left(e^{h c / \lambda K T_{c b}}-1\right)} d \lambda
\end{gathered}
$$

where

$T_{t}$ is the target temperature.

$T_{p}$ is the calibration plate temperature.

$\chi(\lambda)$ is the thermopile' filter spectral transmittance.

$\varepsilon_{p}(\lambda)$ is the calibration plate spectral emissivity.

$\mathcal{E}_{i}$ is the target average emissivity.

$\lambda, K, h$ and $c$ are Planck's law constants.

The radiometer measuring process consists on solving (1) for the physical variable of interest: $\varphi_{t}$ for channel IR3, $T_{t}$ for channels IR2 and IR5, and $\varphi_{t}{ }^{\prime}=\sigma \cdot T_{t}^{+}$obtained using Stefan Boltzmann law from the value of $T_{t}$ for channels IRI and IR4. In order to do that the rest of the variables or constant in (1) has to be measured or subjected to calibration.

\section{B. Sensitivity Quoeficients Analysis}

The main objective of this sensitivity analysis is to obtain a set of equations to relate a change or drift in any of the parameters of (1), that may be affected by noise or uncertainty in the measurement or calibration processes, with an equivalent change in the phy sical variable we want to retrieve. In order to do that, the first step is to take partial derivatives in
(1) with respect to these parameters. Then, we linearized equation (1) around the working point (2):

$$
\begin{gathered}
\Delta V_{\text {out }}=\left|\frac{\partial V_{\text {out }}}{\partial T_{p}}\right|_{T_{p 0}} \cdot \Delta T_{p} \\
\Delta V_{\text {out }}=\left|\frac{\partial V_{\text {out }}}{\partial T_{c b}}\right|_{T_{c b 0}} \cdot \Delta T_{c b} \\
\Delta V_{\text {out }}=\left|\frac{\partial V_{\text {out }}}{\partial\left(T_{c c}-T_{c p}\right)}\right|_{T_{c b 0}} \cdot \Delta\left(T_{c c}-T_{c b}\right) \\
\Delta V_{o u t}=V_{o u t} / S \cdot \Delta S \\
\Delta V_{\text {out }} \approx V_{\text {out }} / F_{s-e} \cdot \Delta F_{s-e} \\
\Delta V_{\text {out }}=\Delta V_{\text {Amp offset_noise }} \\
\Delta V_{\text {out }}=V_{\text {out }} / G \cdot \Delta G
\end{gathered}
$$

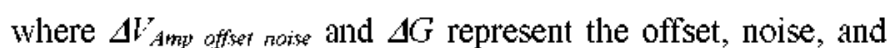
gain errors introduced in thermopiles' readout voltage by the electronics amplification and digitalization chain.

In the same way, a linearized model can be calculated for the phy sical variable of interest (3).

$$
\begin{gathered}
\Delta V_{\text {out }}=\left|\frac{\partial V_{\text {out }}}{\partial T_{t}}\right|_{T_{t 0}} \cdot \Delta T_{t} \\
\Delta V_{\text {out }}=\left|\frac{\partial V_{\text {out }}}{\partial \varphi_{t}}\right|_{\varphi_{t 0}} \cdot \Delta \varphi_{t}
\end{gathered}
$$

In order to convert target temperature variation into equivalent irradiance for channels IRl and IR4, Stefan Boltzmann law has to be differentiated $\Delta \varphi_{t}{ }^{\prime}=4 \cdot \sigma T_{t}{ }^{3} \cdot \Delta T_{t}$. Thus, substituting in (3.1) we have:

$$
\Delta V_{\text {out }}=\left(\left|\frac{\partial V_{\text {out }}}{\partial T_{t}}\right|_{T_{t 0}} / 4 \cdot \sigma \cdot T_{t 0}^{3}\right) \cdot \Delta \varphi_{t}^{\prime}
$$

Equations (3) can be solved for the physical variable of interest, them:

$$
\begin{gathered}
\Delta T_{t}=1 / /\left.\frac{\partial V_{o u t}}{\partial T_{t}}\right|_{T_{t 0}} \cdot \Delta V_{o u t} \\
\Delta \varphi_{t}^{\prime}=4 \cdot \sigma \cdot T_{t 0}^{3} /\left|\frac{\partial V_{o u t}}{\partial T_{t}}\right|_{T_{t 0}} \cdot \Delta V_{\text {out }} \\
\Delta \varphi_{t}=1 / /\left.\frac{\partial V_{o u t}}{\partial \varphi_{t}}\right|_{\varphi_{t 0}} \cdot \Delta V_{\text {out }}
\end{gathered}
$$

Finally, voltage variations from (2) can be converted into the physical variable of interest variations using equations (4). As example. sensitivity to calibration plate temperature measurement errors is provided for the different channels (5):

$$
\Delta T_{t}=\left|\frac{\partial V_{o u t}}{\partial T_{p}}\right|_{T_{p 0}} /\left|\frac{\partial V_{o u t}}{\partial T_{t}}\right|_{T_{t 0}} \cdot \Delta T_{p}
$$




$$
\begin{gathered}
\Delta \varphi_{t}=\left|\frac{\partial V_{o u t}}{\partial T_{p}}\right|_{T_{p 0}} /\left|\frac{\partial V_{o u t}}{\partial \varphi_{t}}\right|_{\varphi_{t 0}} \cdot \Delta T_{p} \\
\Delta \varphi_{t}^{\prime}=4 \cdot \sigma \cdot T_{t 0}^{3} \cdot\left|\frac{\partial V_{o u t}}{\partial T_{p}}\right|_{T_{p 0}} /\left|\frac{\partial V_{o u t}}{\partial T_{t}}\right|_{T_{t 0}} \cdot \Delta T_{p}
\end{gathered}
$$

\section{UNCERTAINTY SOURCES AND VALUES}

Some constants or variables of sensor's mathematical model are foreseen to drift or change during TIRS operational life, or present uncertainty or noise associated to their calibration or measurement processes. In this section reasoned values for these uncertainties and noises at $\mathrm{BoL}$ and $\mathrm{EoL}$ are provided for nominal measurement mode. The figures have been obtained from TIRS thermal model analysis, BB model calibration test results and REMS practical performance and data. Different uncertainties and noise are modeled as independent or uncorrelated gaussian statistical variables, and their shown values have to be understand as one standard deviation [17].

Sensor model parameters directly related to thermopiles are affected by calibration uncertainties and degradation during operations. Thermopiles' responsivity determination, $S$, is altered by calibration set-up errors and ageing because of planetary protection instrument bake-outs, and thermal cycling during Martian operations and cruise phases. These errors are already known from REMS-GTS calibration set-up performance [18], and TIRS thermopiles package qualification and validation (PQV) test results. On the other hand, thermopiles' view factor, $F_{s-e}$, can be degraded as result of dust built up on its filters. In flight calibration algorithm performance in the identification of the degraded view factor is also known from REMS-GTS practical data [10]

During Martian operations the thermopiles will have to operate in a thermally challenging environment. Mars 2020 rover remote sensing mast, where the TIRS is accommodated, is in general hotter than the Martian environment because of the rover thermal control system and its Radioisotope Thermoelectric Generator (RTG) effect. Additionally, Martian ground-atmosphere boundary layer, where TIRS is immersed, is subjected to diurnal and seasonal thermal evolution. This thermal environment is very difficult and costly to recreate inside a simulation chamber. Thus, to estimate thermopiles' package spatial thermal gradients, we have resorted to the thermal model analysis [19], Fig. 2. Fig. 3 provides data for the diurnal evolution of thermopiles' package gradients. The maximum or worst case value $(3 \sigma)$ for the different thermopiles and operational conditions is $0.066 \mathrm{~K}$.

Thermopiles' noise figure is computed from manufacturer datasheet noise spectra density datum [12] and TIRS amplifiers bandwide, which is $150 \mathrm{~Hz}$. Table III summarizes values for thermopiles errors.
TABLE III. THERMOPILES' UNCERTAINTIES $(1 \sigma)$

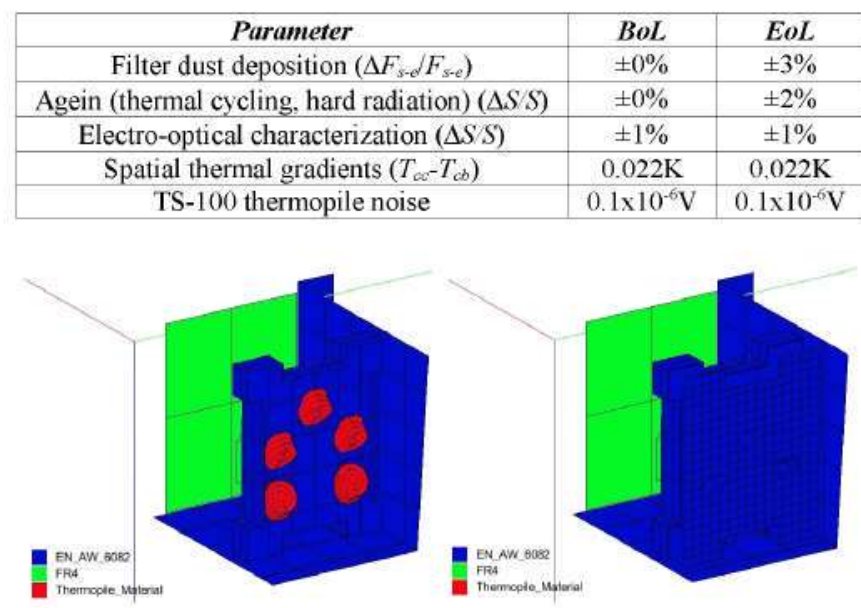

Fig. 2. TIRS thermal model. Support and thermopiles structures (left) and calibration plates structure (right).

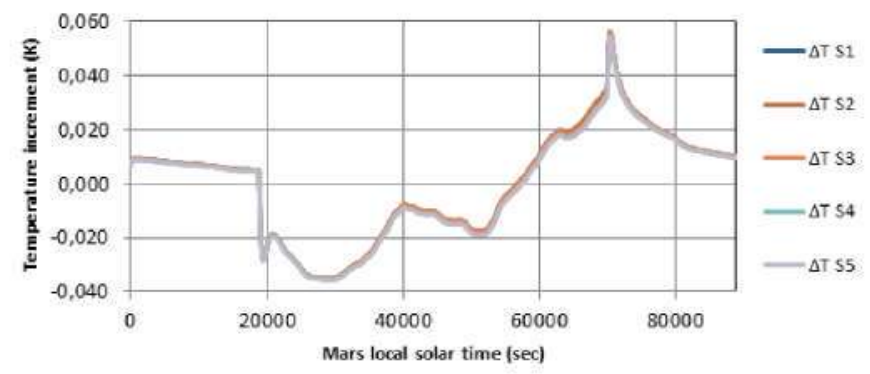

Fig. 3. Diurnal evolution of thermopiles spatial thermal gradients (measured between package lid and socket) from TIRS thermal model worst hot case.

Thermopile's amplification chain, Fig. 4 (top), is based on the ultralow offset and zero drift chopper-stabilized operational amplifier, AD8628. A low frequency offset estimation system based on mechanical relays is also implemented. This system permits to measure thermopile voltage with positive and negative polarity, and in this way limits offset uncertainties to relay contact potential. Amplifier noise figure is mainly dominated by the first stage of the amplification chain.
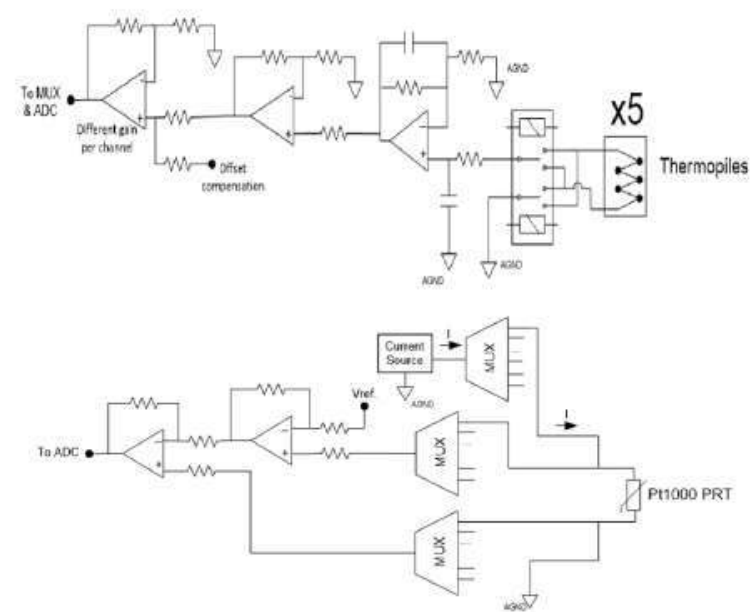

Fig. 4. Thermopiles (top) and RTDs (bottom) electronics conditioning chains 
TABLE IV. AMPLIFICATION CHAIN UNCERTAINTIES AND NOISE (1 $\sigma$ )

\begin{tabular}{|c|c|c|}
\hline Parameter & BoL & EoL \\
\hline Thermopiles amplification chain gain $(\Delta \mathrm{G} / \mathrm{G})$ & $0.22 \%$ & $0.22 \%$ \\
\hline Thermopiles' amplifiers offset $\left(V_{\text {ofset }}\right)$ & $0.67 \times 10^{-6} \mathrm{~V}$ & $0.67 \times 10^{-6} \mathrm{~V}$ \\
\hline RTDs amplifiers offset $\left(T_{c b}, T_{p}\right)$ & $0.077 \mathrm{~K}$ & $0.09 \mathrm{~K}$ \\
\hline Thermopiles' amplifiers noise $\left(V_{\text {noise }}\right)$ & $0.65 \times 10^{-6} \mathrm{~V}$ & $0.65 \times 10^{-6} \mathrm{~V}$ \\
\hline RTDs amplifiers noise $\left(T_{c b}, T_{p}\right)$ & $0.05 \mathrm{~K}$ & $0.05 \mathrm{~K}$ \\
\hline
\end{tabular}

RTD's amplification chain, Fig. 4 (bottom), is based on a multiplexing system, implementing a 4-wire resistance sensing architecture. To compensate offset and gain errors during Martian operations two calibration channels have been included, based on low drift and tolerance resistances as calibration references. Tests at ambient temperature and worst case analysis for the operational temperature range provide the merit figures of Table IV, for thermopiles and RTD amplifiers.

The last set of uncertainties is associated to TIRS calibration and support plate temperatures, Table V. RTDs calibration uncertainty is defined by sensor quality and its calibration method performance using a temperature controlled bath with a platinum resistance pattern. On the other hand there are spatial thermal gradients on support and calibration plates between the RTDs locations and the positions where $T_{c b}$ and $T_{p}$ must be taken for each TIRS channel. We have resorted to TIRS thermal model [19] to estimate these two uncertainties. The diurnal evolution of these gradients for the worst hot case scenario can be seen on Fig. 5 and Fig.6. Newly, maximus values represent 3 standard deviations of the uncertainty.

TABLE V. TIRS'S TEMPERATURES UNCERTAINTIES $(1 \sigma)$

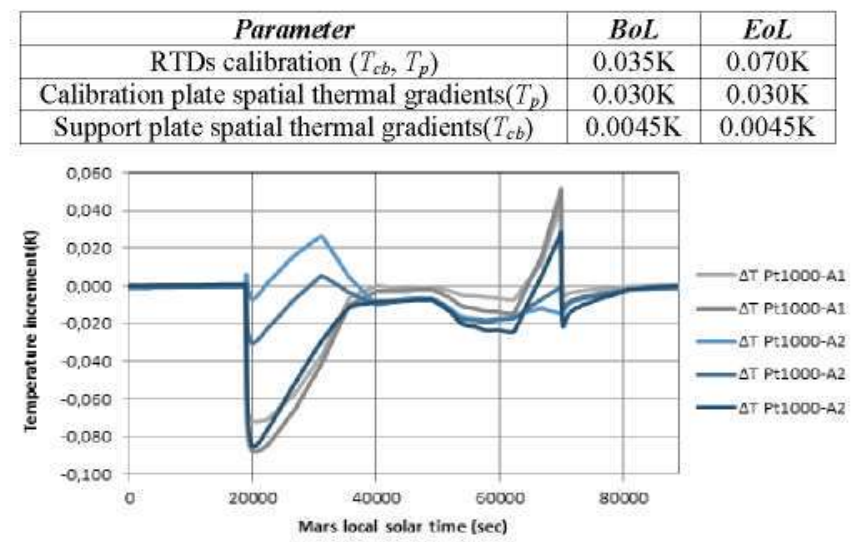

Fig. 5. Diurnal evolution of calibration plate spatial thermal gradients from TIRS thermal model worst hot case.

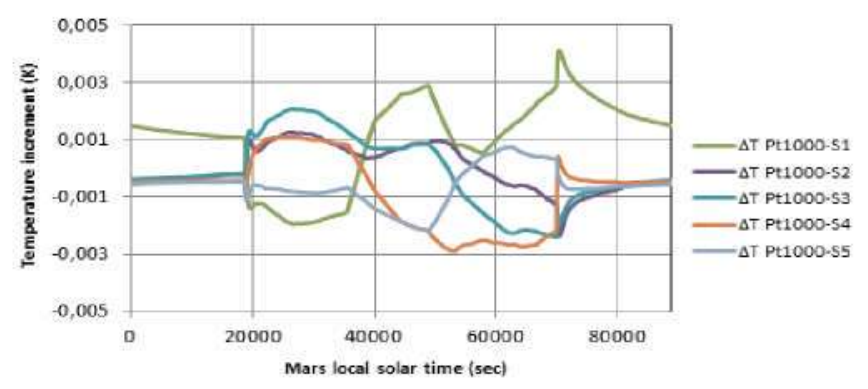

Fig. 6. Diurnal evolution of support plate spatial thermal gradients from TIRS thermal model worst hot case.

\section{TIRS ACCURACY AND RESOLUTION CALCULUS VS. TEMEPRATURE}

In this section, TIRS channels accuracies and resolutions figures are computed. Calculus uses the sensitivity equations of section III and the particular uncertainty and noise contributions of section IV. Finally, different contributions are added using quadratic sum of squares, and therefore assuming uncorrelated error sources.

The calculus is performed considering a nominal scenario for targets and TIRS temperatures and irradiances. Their values have been taken from the hot and cold thermal cases of the Mars 2020 environmental requirements document, Fig. 7. From TIRS thermal model simulations for nominal operational mode, where no power is dissipated on calibration and support plates, it can be said that support and calibration plate temperatures, $T_{c b}$ and $T_{p}$, are almost equal, apart from the described thermal gradients. A summary of the thermal scenario for target temperatures and irradiances is described in Table VI.

Flight lot thermopiles have not been calibrated and assembled yet, so nominal values have been assumed for their corresponding sensor parameters, Table VII. Filters spectral transmittance have been obtained from test using a photo spectrometer [3]. For the mathematical computation of the Plank's law integral inside filter transmission band a sixth order polynomial has been used. The order of the polynomial has been selected to achieve an equivalent temperature fitness error bellow $0.01 \mathrm{~K}$.

TABLE VI. TARGET TEMPERATURE AND IRRADIANCE SCENARIO

\begin{tabular}{|c|c|}
\hline Channel & Target temperature $\left(T_{t}\right)$ or Irradiance $\left(\phi_{s}\right)$ \\
\hline IR1 & $T_{c b}-70 \mathrm{~K}$ \\
\hline IR2 & $T_{c b}-10 \mathrm{~K}$ \\
\hline IR3 & $150 \mathrm{~W} / \mathrm{m}^{2}$ \\
\hline IR4 & $T_{c b}-20 \mathrm{~K}$ \\
\hline IR5 & $T_{c b}-20 \mathrm{~K}$ \\
\hline
\end{tabular}

TABLE VII. THERMOPILES MODEL NOMINAL PARAMETERS VALUES

\begin{tabular}{|c|c|}
\hline Parameter & Value \\
\hline Responsivity $(S)$ & $170 \mathrm{~V} / \mathrm{W}$ \\
\hline Responsivity TC $(S)$ & $-0.47 \% / \mathrm{K}$ \\
\hline Fiev factor $\left(F_{s-e}\right)$ & 0.07 \\
\hline$\alpha$ & 0.45 \\
\hline Thermopiles area & $1 \times 10^{-6} \mathrm{~m}^{2}$ \\
\hline
\end{tabular}

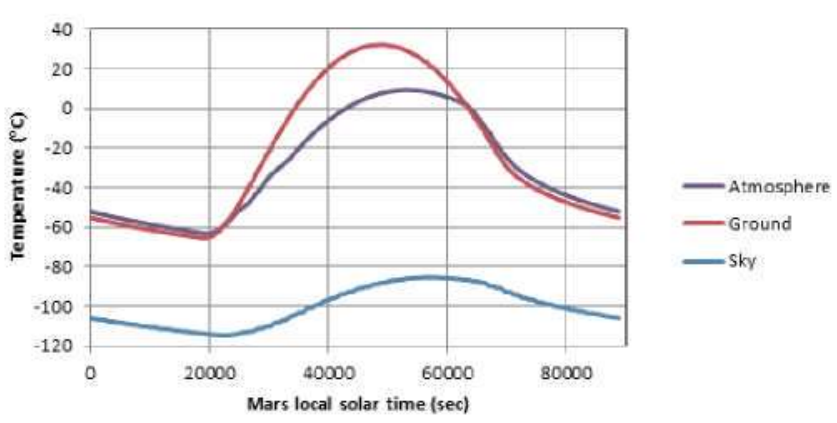

Fig. 7. Target temperatures for the worst hot case conditions. 


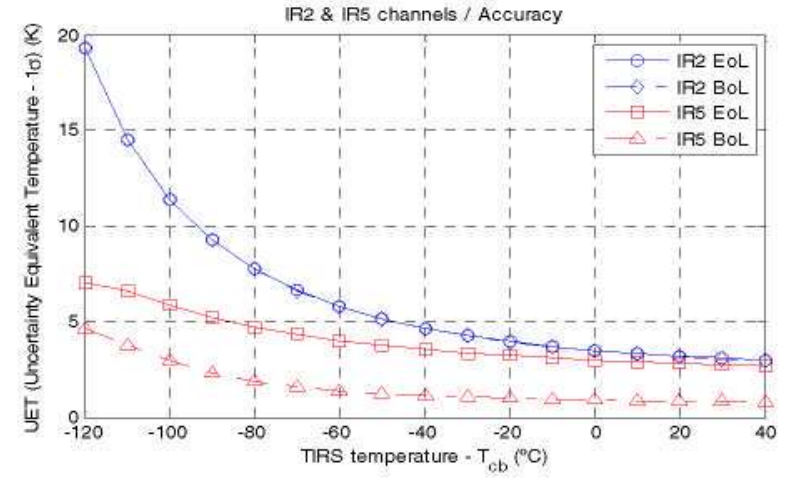

Fig. 8. Channels IR2 and IR5 accuracy at BoL and EoL vs. TIRS temperature.

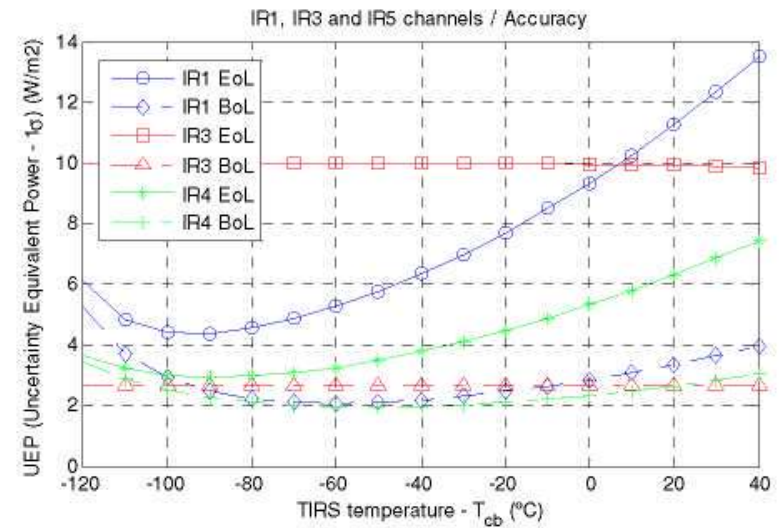

Fig. 9. Channels IR1, IR3 and IR4 accuracy at BoL and EoL vs. TIRS temperature.

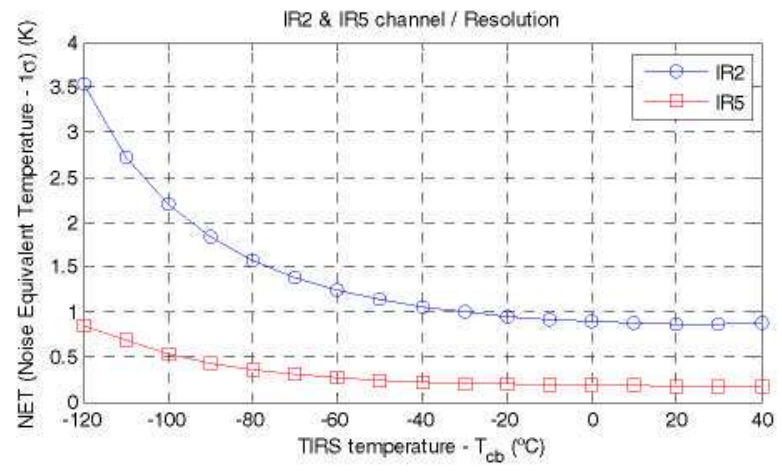

Fig. 10. Channels IR2 and IR5 resolution vs. TIRS temperature.

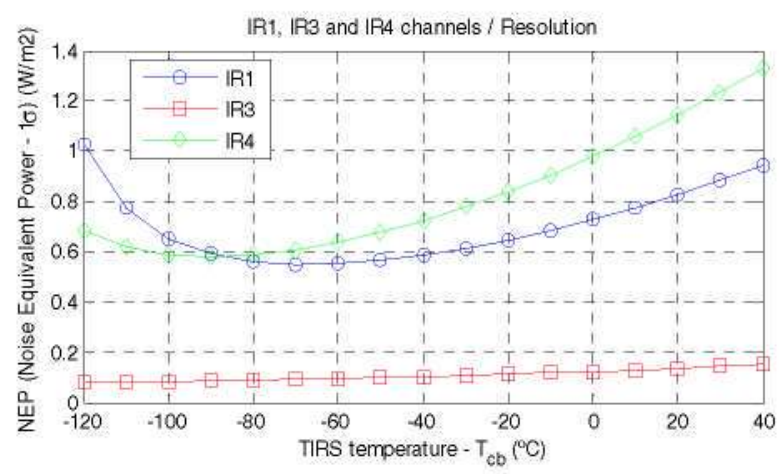

Fig. 11. Channels IR1, IR3 and IR4 resolution vs. TIRS temperature.

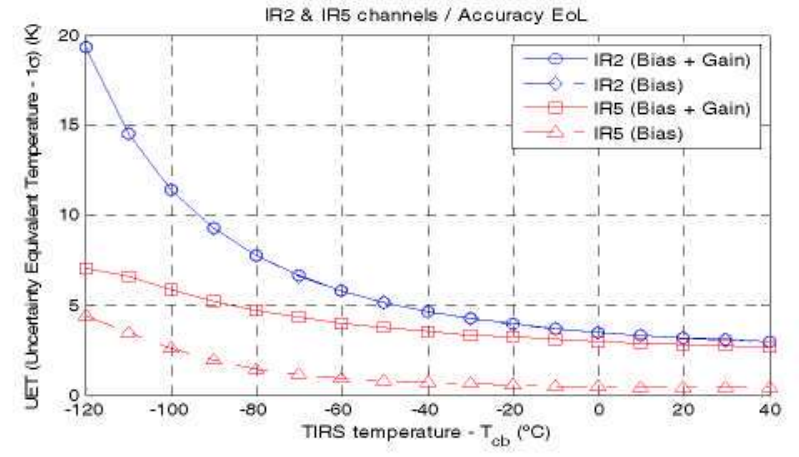

Fig. 12. Channels IR2 and IR5 EoL accuracies vs. TIRS temperature. Total, bias plus gain uncertainties (solid line), bias uncertainties (doted line)

Figures 8 to 11 show TIRS channels expected performance versus temperature, as result of the previously described computation methodology. Accuracies at BoL and EoL, and resolution figures are expressed as one standard deviation $(1 \sigma)$ of the uncertainty or noise equivalent temperature or irradiance, to be directly compared with requirements. As conclusion it can be said that results are compliant with their respective requirements for most of the operational temperatures. Exceptions are channel IR2 at BoL and EoL, and IR5 at EoL for temperatures below $-50^{\circ} \mathrm{C}$ and $-85^{\circ} \mathrm{C}$ respectively.

Figure 12 is dedicated to show separately bias and gain uncertainties contributions to channels IR2 and IR5 accuracies. Differences between channels are easily observable. For channel IR2 bias errors contribution is much more important than gain errors. This fact brings to light the smaller requirements imposed to in-flight calibration algorithm performance for this channel. On the other hand IR5 accuracy is mainly dominated by gain errors, being in-flight calibration algorithm performance an essential factor in the IR 5 accuracy.

Al last, Fig. 13 shows an example of IR3 and IR4 channels' accuracy values due to specific uncertainty sources. Results point out for channel IR3 that support plate's RTD amplifier bias error contribution is small than the uncertainty associated to the thermopiles' spatial thermal gradient all over the operational temperature range. Contrary, IR4 accuracy is clearly determined by support plate's RTD amplifier bias error, especially for the higher operational temperatures. However, for this same channel thermopile's spatial thermal gradient contribution takes on relevance for lower temperatures.

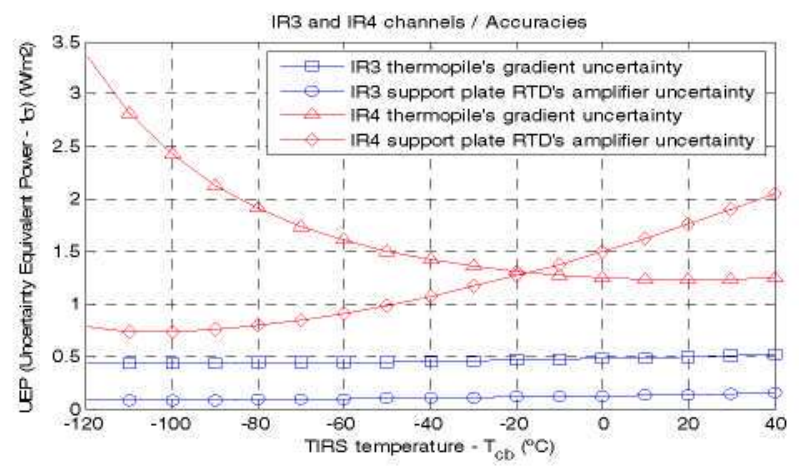

Fig. 13. Channels IR3 and IR4 EoL partial accuracies vs.TIRS temperature. Thermopile spatial thermal gradient uncertainty (solid line), and suport plate RTD amplifier uncertainty (doted line). 


\section{CONCLUSIONS}

The proposed methodology permits to analyze the influence of any particular error source in a radiometer global performance, as well as add all the different sources of error to calculate accuracy and resolution figures. Sensor performance at BoL and EoL can be easily computed, as well as its evolution versus operational temperature. These methodology capabilities are crucial during the radiometer requirement specification and design phases. Another advantage is the possibility of relaxing the complexity of test set-up for functional verification. Partial test results can be introduced in the model and its contribution to global performance can be extrapolated. The necessity of performing complex tests. recreating conditions such as atmosphere composition or rover thermal environment. is therefore partially avoided.

TIRS accuracy and resolution figures are provide for the nominal operational mode. The computation is based on the current best estimate values for uncertainty and noise figures. assuming uncorrelated error sources and gaussian statistical representation for the different variables. In general terms. results show the fulfilment of high level requirements, with some exceptions for specific channels. operational live and temperature. Figures display that each particular uncertainty source exhibits different contributions to accuracy and resolution depending on specific TIRS channel filter transmittance and target temperature. As a general rule. colder targets and narrower thermopiles' bandpass filters imply poorer performances. It is also shown the important contribution to accuracy of thermopiles' spatial thermal gradients.

A deeper analysis of TIRS thermal model results indicates that the diurnal temperature evolution of TIRS support plate is directly transformed in spatial thermal gradients in the thermopile package. This happens as the result of the heat transfer between both elements, through the thermopiles' base, and because of the poor thermal conductivity properties of thermopile's stain-steel package. It is straightforward to infer that thermopiles spatial gradient may be minimized, keeping constant the temperature of the support plate by means of acting on the associated temperature control sy stem in the high performance operational mode. Nevertheless. driving power to support plate heaters creates spatial gradients on support plate and thermopiles' packages. Fortunately, these gradients can be estimated and compensated by means of calibration.

In most of the cases. present error sources and therefore accuracy and resolution figures are considered preliminary. As future work, tests with engineering models of TIRS and amplification electronics will be used to verify accuracy and resolution results for specific test conditions. updating and validating uncertainty source values. This is the case of the error associated to dust deposition, and its compensation by the in-flight calibration algorithm, or the TIRS thermal model predictions that have to include an analysis for the high performance operational mode.

Additionally, the proposed model should be updated to consider a gain error source as result of the uncertainty in the measurement of thermopiles reference temperature, leading to uncertainty in thermopile responsivity. Equally, some error values and model parameters have to be particularized for each channel, e.g. support and calibration plate spatial gradients, thermopiles responsivity or the inflight calibration algorithm performance. Finally, calculus for other target temperature and irradiance scenarios, considering different environmental conditions, such us atmospheric dust opacity, ground thermal inertia, albedo or annual season will be studied too.

\section{ACKNOWLEDGMENT}

This work forms part of the research project regarding the design and scientific issues of the NASA/MARS2020 MEDA. The authors would like to express special thanks to all MEDA Co-Is and personnel who participate in a variety of ways to the development of MEDA TIRS.

\section{REFERENCES}

[1] http:/imars.nasa.govimars2020/mission/instruments/

[2] J.A. Rodrignez-Manfredi et al., "MEDA: An environmental and meteorological package for Mars 2020," Lunar and Planetary Sicience Conference, Texas, vol. 45, pp. 2837, March 2014.

[3] J. Pérez Izquierdo. E. Sebastián Martínez, A. Bravo. R. Ferrándiz. M. Ramos, G. Martinez. and J.A. Rodriguez Manfredi, "The MEDA's Radjometer TIRS for the MARS2020 Mission," AAS/Division for Planetary Sciences Meeting Abstracts, California, vol. 48, November 2016 .

[4] M.D. Smith et al. "Atmospheric science results from the mars exploration rovers Mini.-TES." Science, vol. 306. pp. 1750-1753, 2004.

[5] A.P. Zent et al., "Thermal and Electrical Conductivity Probe (TECP) for Phoenix," J. Geophys. Res, vol. 114, 2009.

[6] J. Gómez-Elvira et al., "REMS: The Environmental Sensor Suite for the Mars Science Laboratory Rover," Space Sci Rev, vol. 170: 583, Augost 2012 .

[7] T. Spobn et al." "Mupus-a thermal and mechanical properties probe for the Rosetta Lander Philae," Space Sci. Rev, vol. 128(1-4), pp. 339-362, 2007.

[8] M. Grott, et al., "The MASCOT Radiometer MARA for the Hayabusa 2 Mission," Space Sci. Rev, pp. 1-26, June 2016.

[9] E. Kopp et al.. "HP3-RAD: A compact radioneter design with on-site calibration for in-situ exploration." Proc. of SPIE Vol. 9973 99730T-8. 2016.

[10] E. Sebastián, C. Armiens, J. Gómez-Elvira. M.P. Zorzano, J. MartinezFrias, B. Esteban, M. Ramos, "The Rover Environmental Monitoring Station Ground Temperature Sensor: A Pyrometer for Measuring Ground Temperature on Mars," Sensors, vol. 10(10), pp. 9211-9231 2010.

[11] M. Grott. J. Knollenberg. A.Maturilli. J. Helbert, N. Müller, E. Kührt, "Mineralogical surface characterization using the MASCOT radioneter MARA on the Hayabusa 2 mission," 44th Lunar and Planetary Science Conference, vol. 1597, 2013.

[12] www,jpht-jena.de, IPHT web page.

[13] C. Escriba, E. Campo, D. Estève, J.Y. Fourniols, "Complete analytical modelling and analysis of micro-machined thermoelectric nncooled IR sensors," Sensor and Actuators A vol. 120. pp. 267-276. 2005.

[14] E. Sebastián. C. Armiens, J.Gómez-Elvira. "Infrared temperature measurement uncertainty for unchopped thermopile in presence of case thermal gradients," Infrared Physics \& Technology, vol. 54, pp. 75-83. 2011.

[15] G. Martínez et al. "Characterization of the modern Martian climate near the surface: A review of in-situ data from Viking to Curiosity". Space Science Reviews, unpublished.

[16] H. Kieffer et al. "Thermal model for analysis of Mars infrared mapping."J. Geophys. Res. Planets, vol. 118. pp. 451-470, 2013. 
[17] BIPM, IEC, IFCC, ISO, IUPAC, IUPAP and OIML (2008) Evaluation of Measurement Data Guide to the Expression of Uncertainty in Measurement JCGM 100:2008 (GUM 1995 with Minor Corrections) lst edo (Sèveses. Paris: BIPM Joint Committee for Guides in Metrology)

[18] E. Sebastian, C. Ammiens, J. Gomez-Elvira, "Pyrometer model based on sensor physical structure and thermal operation," Applied Thermal Engineering, vol. 30 (11). pp. 2403-2411, 2010.

[19] I. Perez-Grande. L. Peinado, A. Chamorro, I. Torralbo, G. Alonso, J. A. Rodriguez Manfredi, A. Lepinette and E. Sebastian, "Thermal design of the Thermal InfraRed Sensor (TIRS) and the Air Temperature Sensor
(ATS) of the Mars Environmental Dynamics Analyzer (MEDA) for Mars $2020^{\circ}$ International Conference on Environmental Systems ICES 2017. Charleston, South Carolina, USA, in press. 